\title{
Unclonable Group Identification
}

\author{
Ivan Damgård, Kasper Dupont, and Michael Østergaard Pedersen
}

Aarhus University, BRICS

\begin{abstract}
We introduce and motivate the concept of unclonable group identification, that provides maximal protection against sharing of identities while still protecting the anonymity of users. We prove that the notion can be realized from any one-way function and suggest a more efficient implementation based on specific assumptions.
\end{abstract}

\section{Introduction}

A large body of literature studies the problem of group identification, where one wants to verify that a given user is a member of a certain group, while ensuring that the user's personal identity is not revealed. Particular instances of this include group signatures $[5,3,22]$ and identity escrow[16]. In some applications, a dishonest user has an interest in giving away to another person the data that allow him to identify himself as a member of the group - such as password and secret keys. The security problems implied by such a scenario have not been given much attention so far in the literature ${ }^{1}$.

In this paper we study this type of problem. As a motivating example, consider the issue of software protection: it is well known that one of the strongest motivating factors in getting people to register as software users is if this enables some functionality that cannot be accessed without registration (and payment). This works particularly well, if the functionality requires access to the vendor's website, since then reverse engineering the software is not sufficient to get unauthorized access to the functionality. In the case of games, for instance, the opportunity to play against others may be available to only registered users, and only through the vendor's website.

Verifying that a user is registered may be done in many different ways. In this paper, we are interested in solutions that work under the following constraints:

- An honest user can connect an unlimited number of times using the same private key material, or at least updates should only be necessary with long time intervals.

- We want to protect users' privacy, i.e., honest users have to identify themselves only as registered users and do not have to reveal their personal identities.

The original version of this chapter was revised: The copyright line was incorrect. This has been corrected. The Erratum to this chapter is available at DOI: 10.1007/978-3-540-34547-3_36

${ }^{1}$ Some earlier works suggest to discourage this by forcing users to either give away all their information, or nothing, but here we are interested in cases where dishonest users in fact have an interest in giving everything away.

S. Vaudenay (Ed.): EUROCRYPT 2006, LNCS 4004, pp. 555-572, 2006.

(C) Springer-Verlag Berlin Heidelberg 2006 
- We want to do as much as possible to protect against attacks where a user "clones" himself by handing a copy of his personal data (software, secret key(s), etc.) to another person in order to get the benefits of two registrations while only paying for one.

Note that the cloning attack may be easy or very hard to carry out physically, depending on how the user's personal keys are stored, but only in very few cases can it be considered impossible.

Of course, we can only hope to detect cloning if the user and clone actually connect to the vendor's website. A further trivial observation is that if first the user connects, then leaves the site and then the clone connects, we cannot distinguish this from two connections made by an honest user, since he would also use the same private key material in both cases. An event we can hope to detect, however, is if both user and clone connect so that they are on the site simultaneously, since this is exactly what cannot occur if the user has been honest. In this case, we not only want to detect the attack, we also want to be able to reveal the identity of the user who cloned himself. Note that, apart from the fact that the above simultaneous scenario is the only one in which we can hope to catch a cloning attack, the scenario is also of practical relevance. For instance, the case of a user who buys one copy of a game and distributes it to all his friends so they can play against each other online, is exactly a case where a number of clones would want to be connected simultaneously.

An unclonable identification scheme informally is an identification scheme where honest users can identify themselves anonymously as members of a group, but where clones of users can be detected and have their identities revealed if they identify themselves simultaneously. In this paper, we give a formal definition of this primitive. We show that it can be realized assuming existence of one-way functions (which is clearly a minimal assumption), and we give a more efficient implementation based on specific assumptions. On the technical side, our most efficient solution is based on a new technique for proving in zero-knowledge, given $g^{x}$ in a group of prime order, that $x$ was chosen pseudorandomly from on a committed secret key.

Of course, before attempting a construction such as we have sketched, one should verify if existing primitives already allow solving the problem. First, one might consider using an anonymous E-cash scheme[17,6], i.e., some number of electronic coins are issued to each user, and users use them to "pay" for access to the site. This would lead to a functionality that is incomparable to the one we sketched above: Cloning in this case means sharing e-coins with others, and so the cloning attack is exactly double spending and can therefore detected even if the two spendings do not take place simultaneously. But on the other hand, honest users can only use each coin once, and must therefore either possess a very large secure memory, or come back for more coins throughout the life of the system. This reveals information on how often a user connects, and is also not consistent with our goal, namely a solution where you can join a group once and then identify yourself an unlimited number of times using the same key material.

One may also consider using group signatures[5, 3,22], and have users identify themselves by signing a message chosen by the verifier (using his current system 
time, for instance). This achieves anonymity but does not protect against cloning. To do this, one would need the property that if the same user signs the same message twice, this would result in signatures that could be detected as coming from the same user. This does not follow from the standard definition of group signatures, and is actually false for known schemes, since these are probabilistic and produce randomly varying signatures even if the message is fixed. A similar comment applies to identity escrow schemes[16].

\section{Definition}

An unclonable identification scheme involves a Group Manager GM, a set of Verifiers and some number of Users. The idea is that after some initialization, there will be several events, where some set of users prove "at the same time" to a verifier $V$ that they are members of the group managed by $G M$. Since we want to detect if $V$ is talking to clones of the same user at the same time, every proof should take as input some string $\alpha$ that represents in some sense the current time or phase of the protocol we are in. However, this does not have to be linked to real time. What is important is that whenever a set of users want to prove themselves, they should agree with $V$ on a value for $\alpha$ that has not been used before. More precisely, the demands are

- An honest $V$ must be able to ensure that all users he talks to at a given point prove themselves using the same value of $\alpha$.

- An honest user should be able to ensure that he never executes Prove with the same value of $\alpha$ more than once.

One solution that works in the case where $V$ runs a website that users would like to be connected to for some length of time, is as follows: with regular intervals, e.g., each hour each user who is connected must prove himself using the current date and hour as $\alpha$, as defined by the verifier's system time. This works if there is sufficient agreement on the time between users and $V$ and if users remember at which time they last did a proof. But many other solutions are possible. Therefore, we have chosen to separate the way time is defined from the definition as such by assuming that the entire system proceeds in consecutive phases, with a unique number assigned to each phase. In each phase, some subset of users decide to prove themselves to some verifier $V$, and the number assigned to the current phase will be used as the string $\alpha$. In the full version of this paper, [10] we propose a way to realize such a scenario without relying on synchronization, or requiring users to keep state.

The system is defined by probabilistic polynomial time algorithms KeyGen, Detect and two-party protocols Join and Prove. These are used as follows:

- Initially, GM runs KeyGen on input $1^{k}$, to get output public key $p k$ and secret key $s k$. We assume for simplicity that the set of possible $p k$ 's output by $\operatorname{KeyGen}\left(1^{k}\right)$ can be recognized in polynomial time.

- When a user $U$ joins the system he runs Join with GM. Common input is $p k$. Private input to $G M$ is $s k$. The protocol outputs to GM either "reject" 
or a string $i d$. Output to $U$ is "reject" or a membership certificate cert $_{U}$. We assume Join is executed on a secure channel so that no other entity will have access to the data exchanged.

- To prove he is a member of the group, the user $U$ executes protocol Prove with a verifier $V$. Common input is the public key $p k$ and the string $\alpha$ assigned to the current phase, $U$ uses cert $_{U}$ as private input. At the end of the protocol $V$ accepts or rejects. Each user executes Prove at most once in every phase.

- Algorithm Detect gets as input a number of transcripts of executions of Prove, done with $p k$ as input in the same phase. It outputs a (possibly empty) list of strings. The intuition is that this algorithm should be able to tell if the result of one or more cloning attacks are among a given set of proofs, and if so, it will output the identities of the involved users.

Definition 1. The algorithms and protocols in a secure unclonable identification scheme must satisfy the following:

Completeness. Assume $G M, V$ and user $U$ are honest. Execution of KeyGen, followed by executions of Join and Prove always result in $V$ accepting.

No Cloning. Consider an honest GM who executes $(p k, s k)=\operatorname{KeyGen}\left(1^{k}\right)$. Consider any probabilistic polynomial time algorithm $\tilde{U}$ who plays the following game on input pk: in any phase, it can issue one or more of the following requests:

1. It can ask that a set of honest users execute Join with GM (no data returned to $\tilde{U})$.

2. It can ask to execute Join itself with GM.

3. It can ask that some number of honest users who already joined the group execute Prove with $\tilde{U}$ acting as verifier, using pk and the current value of $\alpha$ as input.

Finally, $\tilde{U}$ executes Prove a number of times with an honest verifier $V$, on input $p k$ and the current value of $\alpha$.

We now want to capture the idea that in the last step, $\tilde{U}$ can only have proofs accepted by using user identities it got from GM, it must "know" which one of them it is using in each case, and if it uses any of them more then once, the Detect algorithm will catch this.

To this end, we demand that there exists a probabilistic algorithm Extract which gets as input the complete view of $\tilde{U}^{2}$ and outputs a user identity, for every instance of Prove that $V$ accepted in the last step. The expected time to run $\tilde{U}$ and then Extract must be polynomial.

We require that the following holds except with negligible probability: All user identities output by Extract are among those that were generated in the conversations between $\tilde{U}$ and GM. Furthermore, the Detect algorithm, when given as input the conversation between $\tilde{U}$ and $V$, will output exactly those user identities that occur more than once in the output of Extract.

${ }^{2}$ This means that Extract can rewind $\tilde{U}$ to any state that occurred during the game. 
Note that this implies that if $\tilde{U}$ did not execute any Join's, there are no user identities Extract can legally output, so we are then in fact demanding that all $\tilde{U}$ 's proofs are rejected except with negligible probability. Thus we do not need a separate soundness condition in the definition demanding that non-members are rejected.

Anonymity. Consider any probabilistic polynomial time algorithm $\tilde{V}$, who will act as both $G M$ and verifier in an attempt to break the anonymity of honest users. $\tilde{V}$ gets $1^{k}$ as input and outputs a valid pk (can be assumed without loss of generality since we assumed that invalid $p k$ 's can be easily recognized). It then plays the following game: it interacts with a set of honest users, where in each phase some users execute Join and other users execute Prove with $\tilde{V}$. Of course, no honest user will attempt to do Prove unless he already did Join successfully. At some point $\tilde{V}$ stops and outputs a bit, and we let $p_{\text {real }, \tilde{V}}(k)$ be the probability that 1 is output.

We now want to express the demand that $\tilde{V}$ should only learn what is unavoidable, namely the number of honest users that interact with it in each phase. So we compare the above game to a different one, where $\tilde{V}$ interacts with a simulator $M$. The simulator gets as input for each phase the number of users who want to execute Join and the number that want to execute Prove in the current phase. These numbers are chosen with the same distribution as in the first game. Let $p_{\text {sim, } \tilde{V}}(k)$ be the probability that 1 is output in this case.

We demand that there exists a simulator probabilistic polynomial time simulator $M$ such that for any $\tilde{V},\left|p_{\text {real }, \tilde{V}}(k)-p_{\text {sim, } \tilde{V}}(k)\right|$ is negligible in $k$.

We note that in this definition, we have for simplicity used the usual two-phase structure of identification schemes to define soundness and non-cloning, where first the adversary talks to the honest users and then tries to fool the honest verifier. Thus we do not allow him to interact with an honest prover and and honest verifier simultaneously. However, this is not a serious restriction, as there are several techniques that allow handling even this concurrent case, such as the so called designated verifier proofs[12,7]. These techniques can be used with any of the schemes we propose here.

As for the scheduling of the individual protocols in a single phase, we consider two cases: one where in each phase the proofs given to an honest verifier are composed sequentially, and one where the composition may be concurrent, with a scheduling chosen by the adversary. We speak of sequential and concurrent security, accordingly. On the other hand, we assume that honest users (provers) may interact concurrently with an adversarial verifier.

\section{A Theoretical Solution}

\subsection{Some Tools}

We will need a secure string commitment scheme. Such a scheme follows from any one-way function using for instance Naor's construction[18], where there 
is a public key Pcom which is a random string (of length polynomial in the security parameter $k$ ) that can be chosen once and for all by the receiver of commitments. We let $\operatorname{com}_{P \text { com }}\left(s t r, r_{s t r}\right)$ denote a commitment to string str using random coins $r_{s t r}$. Such a commitment determines str uniquely except for a negligible fraction of the public keys, and commitments to different strings are polynomially indistinguishable assuming the underlying one-way function is hard to invert.

Based on such a commitment scheme and, for instance, Blum's protocol for Graph Hamiltonicity or the one from [14] for graph 3-colorability, we can build generic proofs of knowledge for any binary relation $R$ that can be checked in polynomial time. The protocol in its basic form is a three move protocol where the second message is a one-bit challenge from the verifier. When we work with security parameter $k$, we may compose sequentially $k$ instances of this protocol, to obtain a zero-knowledge proof of knowledge for $R$ with negligible soundness error. We may also compose in parallel $k$ instances of the protocol. This is also a proof of knowledge for $R$, more precisely, on common input $x$, the prover proves knowledge of $w$ such that $(x, w) \in R$.

Protocols obtained by this parallel composition are special cases of so-called $\Sigma$-protocols. By definition, such protocols have three properties: first, conversations are of form $(a, e, z)$, where $a=a\left(x, w, \operatorname{coins}_{P}\right)$ is a function of $x, w$ and the prover's random coins, $e$ is a $k$-bit challenge, and $z=z\left(x, w, \operatorname{coins}_{P}, e\right)$ is a function of the prover's private data and the challenge. Based on $x,(a, e, z)$ the verifier decides to accept or reject. Second, the protocol is honest-verifier (computational) zero-knowledge (and is therefore witness indistinguishable). Third, the protocol has the special soundness property, i.e., from $x$ and accepting conversations $(a, e, z),\left(a, e^{\prime}, z^{\prime}\right)$ with $e \neq e^{\prime}$, it is easy to compute $w$ such that $(x, w) \in R$.

Using a technique known as the OR-construction[8], one can combine $\Sigma$ protocols for two relations $R_{0}, R_{1}$, to obtain a new $\Sigma$-protocol, where on input $x_{0}, x_{1}$, the prover proves he knows $w$ such that $\left(x_{0}, w\right) \in R_{0}$ or $\left(x_{1}, w\right) \in$ $R_{1}$, without revealing which is the case, i.e., the protocol is witness indistinguishable.

We will need a family of pseudorandom functions[13]. Such a family is indexed by a key $s$ (a random string of length $k$ bits), and can be designed to have any desired (polynomial in $k$ ) input and output length, assuming any one-way function. We let $f_{s}()$ denote such a pseudorandom function. The basic property is that even given oracle access to the function (and not the key), it cannot be efficiently distinguished from a truly random function.

Finally, we will need a secure signature scheme, which can again be built from any one-way function[21]. Such a scheme comes with probabilistic polynomial time algorithms Gen, Sign, Verify for key generation, signing and verifying signatures. Gen $\left(1^{k}\right)$ outputs a key pair Psign, Ssign. On input message $m$ and the private key, Sign produces a signature $\sigma=\operatorname{Sign}(\operatorname{Ssign}, m)$. On input message, signature and public key, Verify produces as output Verify $($ Psign, $m, \sigma)$ which is accept or reject. 


\subsection{The Scheme}

We first explain the intuition behind the solution: when joining the group, user $U$ will make a commitment $c_{U}$ to a random string $r_{U}$ and will obtain $G M$ 's signature $\sigma_{U}$ on the commitment. He then proves he is a member of the group by proving that he knows a valid signature $\sigma_{U}$ on some message $c_{U}$, without revealing either value. Moreover when giving this proof he uses some random coins. These are not chosen at random but pseudorandomly as $f_{r_{U}}(\alpha)$. That is, he obtains the coins by applying the pseudorandom function to the current $\alpha$-value, using $r_{U}$ as key. He also proves that he has done exactly this. Note that this will force a clone of the user to use the same coins if he gives a proof for the same $\alpha$-value, by security of the commitment and signature schemes. This idea of choosing the randomness for a proof pseudorandomly is somewhat similar to a technique from a completely different context, namely resetable zeroknowledge [15].

The proof given is actually a $\Sigma$-protocol, so the transcripts of proofs given by user and clone are of form $(a, e, z)$ and $\left(a^{\prime}, e^{\prime}, z^{\prime}\right)$. But when all inputs and random coins are the same in the two cases, we must have $a=a^{\prime}$. Furthermore, $e \neq e^{\prime}$ with overwhelming probability, so if both proofs are accepted, special soundness of the protocol means that one can easily compute the prover's secret, which will immediately identify the user in question.

We now describe the components of our scheme - throughout the descriptions, it is understood that a party who detects an invalid proof or signature will immediately stop and reject:

KeyGen. On input $1^{k}$, it generates keys (Psign, Ssign) for the signature scheme and public key Pcom for the commitment scheme (with security parameter $k$ ). Finally, it chooses a random $k$-bit string $R$. The public key is $p k=$ (Psign, Pcom, $R$ ) while the private key is $s k=S$ sign.

Join. The user $U$ sends $c_{U}=$ commit $_{P \text { com }}\left(r_{U}, s_{U}\right)$ where $r_{u}$ is a random $k$-bit string. $G M$ assigns a unique identity $i d_{U}$ to $U$, and sends to $U$ a signature $\sigma_{U}=\operatorname{Sign}\left(\operatorname{Sign},\left(c_{U}, i d_{U}\right)\right)$ on $c_{U}$ concatenated by $i d_{U}$. Also, $G M$ proves in zero-knowledge that he knows a signature (valid under Psign) on $R$. This is easy given that GM knows Ssign. The output certificate for $U$ is $r_{U}, s_{U}, \sigma_{U}, i d_{U}$, while output for $G M$ is $i d_{U}$.

Prove. Recall that $p k$ and the string $\alpha$ is common input to the protocol. User $U$ first makes commitments $C_{U}, D_{U}, E_{U}$ to $c_{u}, i d_{U}, \sigma_{U}$, respectively. He will now give a proof of knowledge related to these commitments, the group public key $p k$ and the number $\alpha$ assigned to the current phase. This proof consists of three ingredients. The first is a proof of knowledge, that $U$ knows how to open the commitments $C_{U}, D_{U}, E_{U}$ to strings $c_{u}, i d_{U}, \sigma_{U}$ such that $\sigma_{U}$ is $G M$ 's signature on $\left(c_{U}, i d_{U}\right)$. While giving this proof, he uses $f_{r_{U}}(\alpha)$ as random coins. That is, the protocol transcript is $\left(a_{1}, e_{1}, z_{1}\right)$, where it should be the case $a_{1}=a_{1}\left(\left(p k, C_{U}, D_{U}, E_{U}\right),\left(c_{U}, i d_{U}, \sigma_{U}\right), f_{r_{U}}(\alpha)\right)$.

The second ingredient is a proof that $U$ can open $C_{U}$ to reveal $c_{U}$ and he knows $s_{U}, r_{U}$ such that $c_{U}=\operatorname{commit}\left(r_{U}, s_{U}\right)$, and the message $a_{1}$ from the 
previous protocol satisfies $a_{1}=a_{1}\left(\left(p k, C_{U}, D_{U}, E_{U}\right),\left(c_{U}, i d_{U}, \sigma_{U}\right), f_{r_{U}}(\alpha)\right)$. Also this proof is a three move protocol of form $\left(a_{2}, e_{2}, z_{2}\right)$, and we are going to do the two proofs in parallel, so that the overall conversation will have form $\left(a_{1}, a_{2}, e_{1}, e_{2}, z_{1}, z_{2}\right)$. The final ingredient is a proof of knowledge of $G M$ 's signature on the string $R$ that is part of $p k$. This is combined with the previous ingredients using the OR construction mentioned above, i.e., $U$ is proving that he knows a signature on $R$, or strings $c_{u}, i d_{U}, \sigma_{U}, r_{U}, s_{U}$ satisfying the conditions just described ${ }^{3}$.

Detect. Looks at all the proofs given in a phase and finds all places where two conversations include tuples of form $\left(a_{1}, a_{2}, e_{1}, e_{2}, z_{1}, z_{2}\right)$, respectively $\left(a_{1}^{\prime} a_{2}^{\prime}, e_{1}^{\prime}, e_{2}^{\prime}, z_{1}^{\prime}, z_{2}^{\prime}\right)$ and where $a_{1}=a_{1}^{\prime}$ and $e_{1} \neq e_{1}^{\prime}$. For any such case it will use the special soundness property to extract the underlying $c_{U}, i d_{U}, \sigma_{U}$, and appends $i d_{U}$ to its output list.

Theorem 1. Assuming one-way functions exist, the above scheme is a secure unclonable identification scheme with sequential security.

We remark that concurrent security can be obtained under the same assumption in the common reference string model, using a technique similar to the one used in the more efficient protocol we describe later.

The key to the proof of the theorem is

Lemma 1. The proof of knowledge given by the user during the Prove protocol is witness indistinguishable

Proof. Recall that the proof given by $U$ is a combination using the OR construction of first a proof of knowledge of a signature on $R$ and second a proof of knowledge of values $c_{u}, i d_{U}, \sigma_{U}, r_{U}, s_{U}$ satisfying a number of properties. Conversations in the latter protocol are of form $\left(a_{1}, a_{2}, e_{1}, e_{2}, z_{1}, z_{2}\right)$. The OR construction leads to a witness-indistinguishable protocol if both protocols used are honest verifier zero-knowledge. This is true for the first protocol, which is just a standard $\Sigma$-protocol and so is honest verifier zero-knowledge by construction.

It is therefore enough to show that the second protocol is honest verifier zeroknowledge. Some notation for this: the part $\left(a_{1}, e_{1}, z_{1}\right)$ of a conversation will be called proof 1 . It has the commitments $C_{U}, D_{U}, E_{U}$ and public key $p k$ as public input, while the secret witness is $c_{U}, i d_{U}, \sigma_{U}$. The rest of the conversation $\left(a_{2}, e_{2}, z_{2}\right)$ is called proof 2. It has $C_{U}, D_{U}, E_{U}, p k, a_{1}$ as public input while the secret witness is $c_{U}, i d_{U}, \sigma_{U}, r_{U}, s_{U}$.

Both proof 1 and proof 2 are $\Sigma$-protocols constructed from generic zeroknowledge techniques as explained above. They therefore have honest verifier simulators $M_{1}, M_{2}$ respectively. However, note that in our context, proof 1 is not done using the normal prover algorithm, we use pseudorandom coins for the prover, and furthermore the key for this pseudorandomness is used as input in proof 2. Hence a proof is required that we can still use $M_{1}, M_{2}$ to simulate. We do this by defining a series of distributions where the first is that of real

${ }^{3}$ Of course, the latter is normally the case, the other option is included for prooftechnical reasons. 
conversations and the last is the one output by the honest verifier simulator we propose. The result will then follow from arguing that each distribution is computationally indistinguishable from the previous one.

The sequence of distributions are produced as follows:

1. Run the honest prover $U$ 's algorithm (with known secret witnesses and random challenges).

2. Same as above, but proof 2 is replaced by running the honest verifier simulator $M_{2}\left(C_{U}, D_{U}, E_{U}, p k, a_{1}\right)$ for proof 2 . Note that this requires that $r_{U}$ is known, to do proof 1 according to the protocol. However, we will still get something indistinguishable from the previous distribution. This is because the output of $M_{2}$ is indistinguishable from a real conversation, even to someone who knows the secret witness for proof 2. Indeed, $M_{2}$ is simulating a protocol constructed from generic techniques based on any commitment scheme as explained earlier. This means that the simulation essentially produces a set of commitments, some of which are opened and some are not. The unopened commitments have contents different from what would be the case in a real conversation, however, this is the only difference. By the hiding property of the commitments, this difference cannot be detected in polynomial time, even knowing what the commitments are supposed to contain.

3. As 2., but the commitment $c_{U}$ is replaced by a commitment to a random value. This is indistinguishable from 2 . by the hiding property of commitments.

4. As 3., but when doing proof 1 , instead of using $r_{U}$ to compute pseudorandom values for the random coins, we use oracle access to the function $f_{r_{U}}()$. We now do not know $r_{u}$ explicitly, but we will produce exactly the same distribution as in 3 .

5. As in 4., but the oracle access to $f_{r_{U}}()$ is replaced by oracle access to a random function. This is indistinguishable from 4 . by pseudorandomness of the function $f_{r_{U}}()$.

6. As in 5., but the transcript of proof 1 is now generated by running the honest verifier simulator $M_{1}$ for proof 1 . This is indistinguishable from 5 ., since there, we ran proof 1 following the prover's normal algorithm, using real random coins. Summarizing, this last distribution is generated by first running $M_{1}\left(C_{U}, D_{U}, E_{U}, p k\right)$ to get $\left(a_{1}, e_{1}, z_{1}\right)$, and running $M_{2}\left(C_{U}, D_{U}, E_{U}, p k, a_{1}\right)$ to get $\left(a_{2}, e_{2}, z_{2}\right)$, and this defines the desired honest verifier simulation.

We can now proceed with the proof of the required properties.

Anonymity: if $\tilde{V}$ behaves such that at least one instance of the Join protocol completes successfully with non-negligible probability, then we can extract from the proof of knowledge given by $\tilde{V}$ a signature on $R$. Note that no attempts to do Prove would occur before this point. Given this signature, it is trivial to simulate (without rewinding) all subsequent instances of Prove knowing only the number of instances to be done in each phase. This cannot be distinguished from the real game by witness indistinguishability of the underlying proofs of knowledge. 
No cloning: we first describe the required Extract algorithm. It will, for each proof $\tilde{U}$ had accepted in the last stage of the attack, rewind $\tilde{U}$ to the start of this proof and try to extract the secret witness it is using by the standard rewinding technique of sending random challenges to $\tilde{U}$ until it answers a new challenge correctly. At this point a valid witness can be extracted. Each such witness must include either a signature on $R$, or a signature $\sigma_{U}$ on a pair of form $\left(c_{U}, i d_{U}\right)$. Extract outputs $i d_{U}$ in the latter case, and a random string in the former. We put the limitation that the algorithm gives up on a proof and outputs a random string if it rewinds more than $2^{k}$ times, where $k$ is the length of challenges.

To estimate the running time of this, note that the probability that $\tilde{U}$ will have a proof accepted, given the state it is in just before the proof, is determined by the number $T$ of challenges it will answer correctly. The probability that we will have to run Extract on the proof is $T 2^{-k}$, while the number of rewinds we have to do is 0 if $T=0,2^{k}$ if $T=1$ and $2^{k} /(T-1)$ if $T>1$. It follows that contribution to the total expected running time from each proof is polynomial. The total expected running time is just the sum of these contributions since we compose sequentially.

To finalize the argument, we need the following

Claim: we may assume that in the output of Extract, we will only see triples $\left(c_{U}, i d_{U}, \sigma_{U}\right)$ that were obtained earlier by $\tilde{U}$ in some instance of Join.

Indeed, if this is false with non-negligible probability, we can break the signature scheme in a chosen message attack: we choose at random to either ask for signatures on all pairs $c_{U}, i d_{U}$ or a signature on $R$ and use this to simulate the Join protocols done by $\tilde{U}$ and all proofs by honest users given to $\tilde{U}$ (without rewinding, we just follow the protocol). Then by witness indistinguishability, $\tilde{U}$ 's behaviour will be essentially the same as before, so the knowledge extraction from $\tilde{U}$ will give us a signature on a new message with non-negligible probability.

Consider now any two Prove instances where the same triple $c_{U}, i d_{U}, \sigma_{U}$ is extracted. Let $\left(a_{1}, a_{2}, e_{1}, e_{2}, z_{1}, z_{2}\right),\left(a_{1}^{\prime}, a_{2}^{\prime}, e_{1}^{\prime}, e_{2}^{\prime}, z_{1}^{\prime}, z_{2}^{\prime}\right)$ be the transcripts of the two instances.

Now, soundness of the Prove protocol implies that we could extract (except with negligible probability) also two ways of opening $c_{U}$ from the two instances, that is, two pairs $\left(r_{U}, s_{U}\right),\left(r_{U}^{\prime}, s_{U}^{\prime}\right)$ such that

$$
c_{U}=\operatorname{commit}_{P \operatorname{com}}\left(r_{U}, s_{U}\right)=\operatorname{commit}_{P c o m}\left(r_{U}^{\prime}, s_{U}^{\prime}\right) \text {, }
$$

and that

$$
a_{1}=a\left(p k,\left(c_{U}, i d_{U}, \sigma_{U}\right), f_{r_{U}}(\alpha)\right), a_{1}^{\prime}=a\left(p k,\left(c_{U}, i d_{U}, \sigma_{U}\right), f_{r_{U}^{\prime}}(\alpha)\right) .
$$

But we must have $r_{U}=r_{U}^{\prime}$, or the the binding property of the commitment scheme is broken. This immediately implies that $a_{1}=a_{1}^{\prime}$, and therefore, since $e_{1} \neq e_{1}^{\prime}$ with overwhelming probability, Detect will successfully extract $i d_{U}$, as required in the definition. 


\section{A More Efficient Solution}

In this section, we present a more efficient unclonable group identification scheme, based on two main ingredients: First a technique recently proposed by Camenisch and Lysyanskaya [3] for digital signatures based on bilinear groups, with protocols for proving knowledge of a signature on a committed value. Second, a new technique for proving that an element in a group is of form $g^{\psi}$ where $\psi$ is a pseudorandom value computed from a committed key. We will borrow some notation from [3] (and several earlier papers): given a public string $x$, a private witness $w$ and a predicate pred,

$$
P K\{w: \operatorname{pred}(x, w)\}
$$

means that we execute a $\Sigma$-protocol for the relation $\{(x, w) \mid \operatorname{pred}(x, w)=\operatorname{true}\}$, that is, a prover convinces a verifier that he knows $w$ such that the predicate on $x$ and $w$ is satisfied. We will also use the following variant:

$$
P K(\kappa)\{w: \operatorname{pred}(x, w)\}
$$

where $\kappa$ is a bit string. This stands for the following: we execute the underlying $\Sigma$-protocol in the normal interactive way, except that the verifier sends as the second message a random string $\kappa$, and the challenge the prover has to answer is determined as $H(x, a, \kappa)$, where $H$ is a hash function, modelled as a random oracle and $a$ is the first message in the original protocol. The point of this construction is that it allows simulation of the protocol without rewinding, due to the "programmability" of the random oracle, and (for the same reason) it also allows knowledge extraction by standard rewinding. Since we will need the last point for the proof, we cannot just use the Fiat-Shamir heuristic.

\subsection{Proofs of Knowledge with Pseudorandom Exponents}

In this subsection, we introduce some tools to be used in our construction. To this end, we consider a group $G_{p}$ of prime order $p$. We will assume $p$ is chosen as a safe prime, i.e., $p=2 q+1$ where $q$ is also prime. $G_{q}$ will denote the (unique) subgroup of $Z_{p}^{*}$ of order $q$.

We further consider the case where a prover knows exponents $x_{1}, \ldots, x_{t} \in Z_{p}$ such that $\beta=\alpha_{1}^{x_{1}} \cdots \alpha_{t}^{x_{t}}$ for publicly known $\beta, \alpha_{1}, \ldots, \alpha_{t} \in G_{p}$. A standard $\Sigma$ protocol for prover $P$ and verifier $V$ can be used to prove knowledge of the $x_{i}$ 's. That is, we want:

$$
P K\left\{\left(x_{1}, \ldots, x_{t}\right): \beta=\alpha_{1}^{x_{1}} \cdots \alpha_{t}^{x_{t}}\right\}
$$

This $\Sigma$-protocol works as follows:

1. $P$ chooses $r_{1}, \ldots, r_{t} \in Z_{p}$ uniformly at random and sends to $V \tau=\prod_{i=1}^{t} \alpha_{i}^{r_{i}}$.

2. $V$ chooses a random challenge $\epsilon \in Z_{p}$.

3. $P$ responds with $z_{i}=r_{i}+\epsilon x_{i} \bmod p$ for $i=1$..t. $V$ checks that $\prod_{i=1}^{t} \alpha_{i}^{z_{i}}=$ $\tau \beta^{\epsilon}$. 
It is well known (and straightforward to show) that this protocol is indeed a $\Sigma$ protocol for the underlying relation. It is also well-known that a straightforward variant of the protocol allows us to do the following type of proof:

$$
P K\left\{\left(x_{1}, . ., x_{t}, x_{1}^{\prime}, \ldots, x_{t}^{\prime}\right): \beta=\alpha_{1}^{x_{1}} \cdots \alpha_{t}^{x_{t}}, \beta^{\prime}=\alpha_{1}^{\prime x^{\prime}{ }_{1}} \cdots \alpha_{t}^{\prime x^{\prime}}{ }_{t}, x_{1}=x_{1}^{\prime}\right\}
$$

Basically, we run the original protocol twice in parallel for the two equations. This would normally involve two independent sets of random numbers $r_{1}, \ldots, r_{t}$ and $r_{1}^{\prime}, \ldots, r_{t}^{\prime}$. However, to demonstrate that $x_{1}=x_{1}^{\prime}$ the prover must use $r_{1}=r_{1}^{\prime}$ and the verifier checks that the responses $z_{1}, \ldots, z_{t}, z_{1}^{\prime}, \ldots z_{t}^{\prime}$ satisfy $z_{1}=z_{1}^{\prime}$. We will use this variant later, but for now we stick to the basic version for simplicity. All techniques we describe here can also be applied to the variant in a straightforward way.

We now consider a change to the protocol where $P$ chooses the randomness in the first message according to a pseudorandom function $\Psi_{K}(i, \alpha, b)$, where $K$ is a key committed to by $P, \alpha$ is a public input, $i$ is a number and $b$ is a bit. We will use a variant of the pseudorandom function of Naor and Reingold, based on the DDH assumption in $G_{q}$, so that outputs from $\Psi$ are in $G_{q}$. We specify below how the function works and how the key is committed. However, in the previous protocol, the random exponents were chosen in $Z_{p}$, whereas the pseudorandom function produces output in the subgroup $G_{q}$. To resolve this, we let the exponents be chosen as the difference between two pseudorandom values, which allows us to hit all of $Z_{p}$. The modified protocol then works as follows:

1. $P$ sets $r_{i}=\Psi_{K}(i, \alpha, 0)$ and $s_{i}=\Psi_{K}(i, \alpha, 1)$ and sends to $V \tau=\prod_{i=1}^{t} \alpha_{i}^{r_{i}-s_{i}}$.

2. $V$ chooses a random challenge $\epsilon \in Z_{p}$.

3. $P$ responds with $z_{i}=r_{i}-s_{i}+\epsilon x_{i} \bmod p$ for $i=1$..t. $V$ checks that $\prod_{i=1}^{t} \alpha_{i}^{z_{i}}=$ $\tau \beta^{\epsilon}$.

To argue that this is a $\Sigma$-protocol for the same relation, we need a result by Perron[20]: Let $Q R_{p}$ be the set of quadratic residues $\bmod p$. Then for any $a \in Z_{p} *$, the set $a+Q R_{p}$ contains almost as many quadratic residues as nonresidues: the difference is at most 1 . Since in our case $G_{q}=Q R_{p}$, we get from this:

Lemma 2. The distribution of $u_{i}-v_{i} \bmod p$ where $u_{i}, v_{i}$ are chosen uniformly in $G_{q}$, is statistically close to uniform over $Z_{p}$.

Lemma 3. Under the DDH assumption in $G_{q}$, the above protocol is a $\Sigma$-protocol for the relation specified in (1).

Proof. Completeness is trivial, and special soundness follows exactly as for the previous standard protocol. For honest verifier zero-knowledge, we argue as follows: To simulate, we will choose $\epsilon$ and $z_{i}$ at random in their respective domains and then set $\tau=\beta^{-\epsilon} \prod_{i=1}^{t} \alpha_{i}^{z_{i}}$.

Now, assuming $K$ is known only to $P$, pseudorandomness of $\Psi$ implies that our variant is indistinguishable from a protocol where $\Psi_{K}(i, \alpha, 0), \Psi_{K}(i, \alpha, 1)$ are replaced by uniformly random choice $u_{i}, v_{i}$ from $G_{q}$. This creates a distribution of $z_{i}$ that is statistically close to the simulated distribution by Lemma 2 . 
Our goal is now to allow $P$ to prove that he has followed the specified algorithm for choosing the $r_{i}, s_{i}$ 's pseudorandomly. The first step of this is to have $P$ commit to each individual value under a public key chosen by a third party (which will eventually be the group manager in our case). The public key will be two random elements $\eta, \lambda \in G_{p}$, and $P$ will make commitments $\operatorname{com}_{i}=\eta^{r_{i}} \lambda^{\omega_{i}}$ and $\operatorname{com}_{i}^{\prime}=\eta^{s_{i}} \lambda^{\omega_{i}^{\prime}}$, for $i=1 . . t$ and random $\omega_{i}, \omega_{i}^{\prime}$. We can now ask $P$ to prove that he committed to the correct values, that is, execute

$$
\begin{aligned}
P K\left\{\left(r_{i}, s_{i}, \omega_{i}, \omega_{i}^{\prime}, i=1 . . t\right): \tau\right. & =\prod_{i=1}^{t} \alpha^{r_{i}}\left(\alpha^{-1}\right)^{s_{i}}, \\
\operatorname{com}_{i} & \left.=\eta^{r_{i}} \lambda^{\omega_{i}}, \operatorname{com}_{i}^{\prime}=\eta^{s_{i}} \lambda^{\omega_{i}^{\prime}}, i=1 . . t\right\}
\end{aligned}
$$

The $\Sigma$-protocol for this is a standard variant of the one we presented above.

The final step is to show that each committed value was chosen according to the pseudorandom function. For this, we need to specify in detail how it works. We assume that input strings to $\Psi$ all have length at most $k$ (where $k$ can in principle be arbitrary). A key to the function is a number $K \in Z_{q}$. Finally, we will need a hash function $H$ that take a string str of length at most $k$ as input and outputs an element in $G_{q}$. We will model this function as a random oracle. The pseudorandom function is now defined as:

$$
\Psi_{K}(s t r)=H(s t r)^{K} \bmod p
$$

We note that the function mapping $y$ to $y^{K} \bmod p$ is a weak pseudorandom function assuming the DDH assumption holds in $G_{q}$, i.e., as long as $y$ is randomly chosen and is not controlled by the adversary, the outputs look random. However, in our case, and assuming the random oracle model, the function is only used on values produced by $H$, and these are guaranteed to be random, even if the adversary chooses the inputs to $H$. This argument is easily formalized to prove.

Lemma 4. In the random oracle model, and assuming DDH holds in $G_{q}, \Psi_{K}()$ as defined above is a strong pseudorandom function.

We will assume that the key $K$ is committed to by $P$ in a somewhat nonstandard way which, however, fits nicely with the construction we will see in the following. Concretely, we assume that $d=g^{\gamma^{K}} \delta^{r} h^{u}$ is given, for publicly known $g, h \in G_{p}$ and $\gamma, \delta \in G_{q}$. With this, we can summarize our goal, namely to give a $\Sigma$-protocol implementing

$$
\begin{aligned}
P K\left\{\left(K, r, u, \omega_{i}, \omega_{i}^{\prime}, i=1 . . t\right): d\right. & =g^{\gamma^{K} \delta^{r}} h^{u}, \\
\operatorname{com}_{i} & \left.=\eta^{\Psi_{K}(i, \alpha, 0)} \lambda^{\omega_{i}}, \operatorname{com}_{i}^{\prime}=\eta^{\Psi_{K}(i, \alpha, 1)} \lambda^{\omega_{i}^{\prime}}, i=1 . . t\right\}
\end{aligned}
$$

For this, it will be be enough to show how $P$ can prove that some given commitment com satisfies com $=\eta^{\Psi_{K}(s t r)} \lambda^{\omega}$ for public str. Since anyone can compute $\psi=H($ str $)$, our task reduces to:

$$
P K\left\{(K, r, u, \omega): d=g^{\gamma^{K} \delta^{r}} h^{u}, \operatorname{com}=\eta^{\psi^{K}} \lambda^{\omega}\right\}
$$


A protocol for this follows here:

1. $P$ chooses $s, w \in Z_{q}, \nu, \phi \in Z_{p}$ at random. He sends $v_{1}=g^{\gamma^{s} \delta^{w}} h^{\nu}$ and $v_{2}=\eta^{\psi^{s}} \lambda^{\phi}$ to $V$.

2. $V$ selects a random bit $c$.

3. $P$ sends $z_{1}=s-c K \bmod q, z_{2}=w-c r \bmod q, z_{3}=\nu-c u \gamma^{s-K} \delta^{w-r} \bmod p$, and $z_{4}=\phi-c \omega \psi^{s-K} \bmod p$.

$V$ checks as follows: if $c=0$, that $g^{\gamma^{z_{1}} \delta^{z_{2}}} h^{z_{3}}=v_{1}$ and $\eta^{\psi^{z_{1}}} \lambda^{z_{4}}=v_{2}$. If $c=1$,

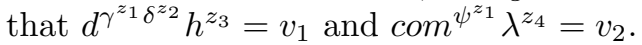

Since this protocol only works with a 1-bit challenge, we need to repeat it an appropriate number of times to have a sufficiently small soundness error.

Lemma 5. The above is a $\Sigma$-protocol for the relation specified in (2)

Proof. Completeness follows by inspection of the protocol. Special soundness: if for given $v_{1}, v_{2}$, the prover can send satisfactory answers $z_{1}, z_{2}, z_{3}, z_{4}$ to $c=0$ and $z_{1}^{\prime}, z_{2}^{\prime}, z_{3}^{\prime}, z_{4}^{\prime}$ to $c=1$, we have by the checks carried out by $V$ that $g^{\gamma^{z_{1}}} \delta^{z_{2}} h^{z_{3}}=v_{1}$,

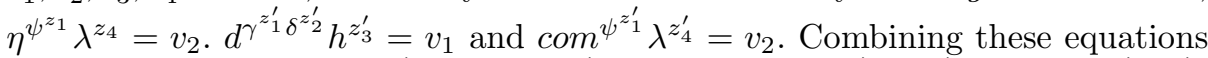
imply that com $=\eta^{\Psi^{z_{1}-z_{1}^{\prime}}} \lambda^{\left(z_{4}-z_{4}^{\prime}\right) \psi^{-z_{1}^{\prime}}}$ and $d=\alpha^{\gamma^{z_{1}-z_{1}^{\prime}} \delta^{z_{2}-z_{2}^{\prime}}} h^{\left(z_{3}-z_{3}^{\prime}\right) \gamma^{-z_{1}^{\prime}} \delta^{-z_{2}^{\prime}}}$, i.e., $a, d$ are of the required form. Finally, honest verifier ZK is argued by the following simulator: choose $z_{1}, z_{2}$ at random in $Z_{q}, z_{3}, z_{4}$ at random in $Z_{p}$ and $c$ as a random bit. If $c=0$, set $v_{1}=g^{\gamma^{z_{1}}} \delta^{z_{2}} h^{z_{3}}$ and $v_{2}=\eta^{\psi^{z_{1}}} \lambda^{z_{4}}$. If $c=1$, set

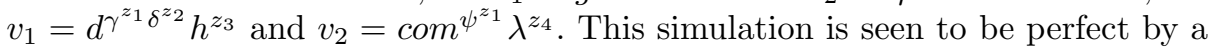
standard argument.

\subsection{The New Scheme}

Our main idea for the scheme is similar to the earlier theoretical one: the user $U$ will commit to a secret key $K$. When registering with the group manager $G M$ he will obtain a signature on the commitment $c_{U}$, using the signature system described in [3] (called scheme A in [3]). He can now prove membership of the group by proving knowledge of a valid signature on $c_{U}$ (as well as proving knowledge of this value). If he tries to clone his identity we can exploit the special soundness property of the protocol used and extract his identity.

KeyGen. Let $G M$ take a security parameter $k$ and output two groups $G_{p}=\langle g\rangle$ and $\mathbf{G}_{\mathbf{p}}=\langle\mathbf{g}\rangle$ of prime order $p=\Theta\left(2^{k}\right)$ where $p=2 q+1$ and $q$ is a prime. Let $G_{q}$ denote the unique subgroup of $Z_{p}^{*}$ of order $q$. Let $\gamma, \delta$ be random generators of $G_{q}$. Let $e: G_{p} \times G_{p} \rightarrow \mathbf{G}_{\mathbf{p}}$ be an efficiently computable bilinear map, and $\eta, \lambda$ be random generators of $\mathbf{G}_{\mathbf{p}}$.

To set up the signature scheme, $G M$ chooses the following values at random: $x \in Z_{p}, y \in Z_{p}$ and sets $X=g^{x}, Y=g^{y}$. The secret key for the signature scheme is $S_{k}=(x, y)$ and the public key is $P_{k}=\left(q, G_{p}, \mathbf{G}_{\mathbf{p}}, g, \mathbf{g}, e, X, Y, \eta, \lambda\right)$. Join. The user $U$ chooses at random $r_{U} \in Z_{q}$ and a key $K \in Z_{q}$. U makes a commitment $c_{U}=\gamma^{K} \delta^{r_{U}} \bmod p$ to $K$ and sends it to $G M^{4}$.

\footnotetext{
${ }^{4}$ It is not necessary to have $U$ prove that he can open $c_{U}$, since later in the Prove
} protocol, he must implicitly show he can open it to have the proof accepted. 
$G M$ verifies that $U$ is allowed to join the group and if so, he computes a signature $\sigma=(a, b, c)$ on $c_{U}$ where $a$ is chosen at random in $G_{p}, b=a^{y}$, $c=a^{x+c_{U} x y}$ and sends it to $U$. GM considers $c_{U}$ as the user's id in the following, whereas $\left(K, r_{U}, a, b, c\right)$ serves as the membership certificate.

Prove. Recall that the string $\alpha$, denoting "the current time", is common input to $U$ and $V . U$ essentially proves that he is a member of a group by proving that he knows a valid message and signature from $G M$. First $U$ blinds his signature $\sigma$ by choosing at random $\mu, r^{\prime} \in Z_{p}$ and computing $\widetilde{\sigma}=(\widetilde{a}, \widetilde{b}, \hat{c})$ where $\widetilde{a}=a^{r^{\prime}}$, $\widetilde{b}=b^{r^{\prime}}, \hat{c}=\left(c^{r^{\prime}}\right)^{\mu}$. Then $U$ makes a commitment $C_{U}=\eta^{c_{U}} \lambda^{s_{U}}$ for random $s_{U}$ and sends $\widetilde{\sigma}$ and $C_{U}$ to $V$. Both compute

$$
v_{x}=e(X, \widetilde{a}), \quad v_{x y}=e(X, \widetilde{b}), \quad v_{s}=e(g, \hat{c})
$$

$V$ chooses a $k$-bit string $\kappa$ at random, and $U$ proves knowledge of a signature on $c_{U}$ to $V$ by giving the following proof:

$$
P K(\kappa)\left\{\left(c_{U}, \rho, s_{U}\right): C_{U}=\eta^{c_{U}} \lambda^{s_{U}}, v_{s}{ }^{\rho}=v_{x} v_{x y}^{c_{U}}\right\}
$$

Here, as $\rho$, the honest $U$ uses $\rho=\mu^{-1} \bmod p . V$ will accept if this proof is correct and it holds that:

$$
e(\widetilde{a}, Y)=e(g, \widetilde{b})
$$

Note that it was shown in [3] that the checks carried out by $V$ plus the proof that $v_{s}{ }^{\rho}=v_{x} v_{x y}^{c_{U}}$ together imply that $U$ must know a valid signature on a message. Doing the proof in (3) is a straightforward variant of the general type of proof from lemma 3 as discussed earlier. Consider the underlying $\Sigma$-protocol for the part relating to $v_{s}{ }^{\rho}=v_{x} v_{x y}^{c_{U}}$. After specializing it to the concrete scenario here, it will have a first message of form

$$
\tau=v_{x y}^{r_{1}-s_{1}} v_{s}^{r_{2}-s_{2}}
$$

Furthermore, we will require that

$$
r_{1}=\Psi_{K}(1, \alpha, 0), r_{2}=\Psi_{K}(2, \alpha, 0), s_{1}=\Psi_{K}(1, \alpha, 1), s_{2}=\Psi_{K}(2, \alpha, 1)
$$

$U$ must therefore prove that the values of $r_{1}, r_{2}$ and $s_{1}, s_{2}$ were generated pseudorandomly from $K$. As described earlier, $U$ does this by making commitments

$$
\operatorname{com}_{1}=\eta^{r_{1}} \lambda^{\omega_{1}}, \operatorname{com}_{2}=\eta^{r_{2}} \lambda^{\omega_{2}}, \operatorname{com}_{1}^{\prime}=\eta^{s_{1}} \lambda^{\omega_{1}^{\prime}}, \operatorname{com}_{2}^{\prime}=\eta^{s_{2}} \lambda^{\omega_{2}^{\prime}},
$$

proving that these values are correct with respect to $\tau$ and finally using the protocol from Lemma 5 to show that each commitment contains a pseudorandom value of correct form computed from $K$ as committed to by $C_{U}$. Note that $C_{U}$ is in fact a commitment to $K$ of exactly the form needed for that protocol.

All proofs to be given during Prove can be done simultaneously, using the same challenge in all $\Sigma$-protocols.

The amount of computation required during Prove is $57+68 r$ exponentiations and 8 evaluations of the function $e$, where $r$ is the number of times the protocol 
from Lemma 5 is repeated. Prove also requires $29 k+r(6 k+1)$ bits sent between $U$ and $G M$, where $r$ is the same as before and $k$ is the security parameter. For example with $r=16$ and $k=1024$, Prove requires approximately 1150 exponentiations and needs to communicate $130 \mathrm{~KB}$. It would be interesting to find a protocol that solves the same problem using a constant number of exponentiations and communication.

Detect. Look at all proofs given in a phase and find all places where two conversations include first messages $\tau, \tau^{\prime}$ where $\tau=\tau^{\prime}$. If the two challenge values involved in these two conversations are different, use the special soundness property to extract a witness for the proof in question - this will be a pair of form $\left(c_{U}, \rho\right)$. Output all $c_{U}$ 's found this way.

Theorem 2. Assuming security of the signature scheme from [3], the DDH assumption in $G_{q}$, and Assumption 1, the scheme described above is a secure unclonable identification scheme in the random oracle model, with sequential security. The Join and Prove protocols are constant-round, and have communication complexity $O(k)$ bits, respectively $O\left(k^{2}\right)$ bits.

The scheme described here is extremely similar in structure to the theoretical solution we gave earlier, so the proof is very similar as well. We only sketch it here. Completeness follows by inspection of the protocols. For no cloning, the required Extract algorithm will use standard rewinding to extract witnesses for all proofs given. By a standard argument, this will succeed for all proofs that were accepted by the verifier, with overwhelming probability. Soundness of the proofs means we will extract a set of user id's and corresponding signatures, so security of the signature scheme implies that this forms a subset of the user id's defined in previous Join protocols. Now, soundness of the proofs from Lemma 5 and the binding property of the commitment schemes defined by $(\gamma, \delta),(\eta, \lambda)$ imply that the adversary must have used the key involved correctly and consistently, and hence the value of $\tau$ will be identical in all instances of subproof (3), where the same key was used. This allows Detect to recover the required information. As for anonymity, note that all subproofs except the one from (3) can be replaced by (perfect) simulations without changing the view of the adversary. After this change, the key $K$ is only used to call the pseudorandom function, and no other information on $K$ is present, since the commitment $c_{U}$ hides $K$ perfectly. We can therefore use Lemma 3 to conclude that also instances of subproofs from (3) can be replaced by simulations without this being detectable by the adversary.

\section{On Concurrent Security}

For both the theoretical and the more efficient solution, it holds that all the proofs given by honest users can be simulated without rewinding. Hence, the only problem in obtaining concurrent security lies in the Extract algorithm that is required for the no cloning property, and which requires rewinding in both solutions.

To avoid this, we can use, at a small efficiency cost, the technique of Fischlin [11], which shows how to transform any $\Sigma$-protocol in the random oracle model 
into a new one for which there is an on-line extractor, i.e., one can extract the secret witness from a successful prover without rewinding.

Using this transformation on the $\Sigma$-protocols underlying our Prove-protocol immediately gives a concurrently secure solution.

\section{On Membership Revocation and Framing}

After discovering the identity of a dishonest user, the group manager needs to act. In some applications it may be sufficient to take some appropriate, say, legal action against the user in question. But it may also be necessary to remove the user out from the group by ensuring that the value $c_{U}$ can never be used again.

Since the value $c_{U}$ is unconditionally hidden in the Prove protocol, nothing in the above systems prevents a dishonest user from proving membership of the group again at a later point in time. To allow for revocation of memberships, we can extend the protocol with an dynamic accumulator as described in [4]. An accumulator scheme $[1,2]$ is an algorithm that allows one to hash large set of values into a short value, called the accumulator such that there is a witness that a given input is in the accumulator. A dynamic accumulator allows one to efficiently add and remove values from the accumulator. It can be used in the following way.

When the user joins the group and sends $c_{U}$, the group manager adds $c_{U}$ to the accumulator. To prove membership of the group, the user is now required, in addition to the protocol we already have, to prove that the value $c_{U}$ is in the accumulator. We will omit the details of how this is done, they can be found in [4]. The solutions needs that $c_{U}$ is committed to, but this is already done in our protocol.

When the identity of a dishonest user is discovered, the group manager removes $c_{U}$ from the accumulator, which prevents the user or any clones of the user from proving membership of the group, as long as the verifier is aware of the new accumulator value.

An aspect we have not been concerned with in this paper is whether the group manager can frame an honest user, that is, create on his own a protocol transcript where the user seems to have cloned himself. For our efficient solution, we believe framing is not possible, since the group manager does not know the user's secret key $K$. The part of the Prove protocol where the user proves knowledge of $K$ can only be simulated without knowing $K$ if one can control the outputs of the random oracle. While we use this to show zero-knowledge in the theoretical analysis, such control is not available to anyone in real life.

\section{References}

1. Josh Benaloh, Michael de Mare: One-Way Accumulators: A Decentralized Alternative To Digital Signatures, proc. of EUROCRYPT 1993.

2. Josh Benaloh, Michael de Mare: Collision-free accumulators and fail-stop signature schemes without trees, proc. of EUROCRYPT 1997.

3. J. Camenisch, A. Lysyanskaya: Signature Schemes and Anonymous Credentials from Bilinear Maps, Proc. of Crypto 04, Springer Verlag LNCS 3152. 
4. J. Camenisch, A. Lysyanskaya: Dynamic Accumulators and Application to Efficient Revocation of Anonymous Credentials, Proc. of Crypto 02, Springer Verlag LNCS 2442 .

5. G.Ateniese, J.Camenisch, M.Joye, G.Tsudik: A practical and provably group signature scheme, Proc. of Crypto 00, Springer Verlag LNCS 1880.

6. S.Brands: Untraceable Off-line Cash in Wallets with Observers, proc. of Crypto 93.

7. Ronald Cramer, Ivan Damgård: Fast and Secure Immunization Against Adaptive Man-in-the-Middle Impersonation. EUROCRYPT 1997: 75-87

8. Ronald Cramer, Ivan Damgård, Berry Schoenmakers: Proofs of Partial Knowledge and Simplified Design of Witness Hiding Protocols, CRYPTO 1994: 174-187

9. Ivan Damgård, Mads Jurik: Client/Server Tradeoffs for Online Elections. Public Key Cryptography 2002: 125-140

10. Damgård, Dupont and Pedersen: Unclonable Group Identification the Eprint archive, www.iacr.org.

11. M.Fischlin: Communication-Efficient Non-Interactive Proofs of Knowledge, proc. of Crypto 2005, Springer Verlag LNCS 3621.

12. Markus Jakobsson, Kazue Sako, Russell Impagliazzo: Designated Verifier Proofs and Their Applications. EUROCRYPT 1996: 143-154.

13. Oded Goldreich, Shafi Goldwasser, Silvio Micali: How to Construct Random Functions, FOCS 1984: 464-479.

14. Oded Goldreich, Silvio Micali, Avi Wigderson: Proofs that Yield Nothing But Their Validity or All Languages in NP Have Zero-Knowledge Proof Systems J. ACM 38(3): 691-729 (1991).

15. Ran Canetti, Oded Goldreich, Shafi Goldwasser, Silvio Micali: Resettable zeroknowledge (extended abstract). STOC 2000: 235-244

16. J.Kilian and E.Petrank: Identity Escrow, Proc. of Crypto 98.

17. D.Chaum, A.Fiat, M.Naor: Untraceable Electronic Cash, proc. of CRYPTO 88.

18. Moni Naor Bit Commitment Using Pseudorandomness, J. Cryptology 4(2): 151-158 (1991).

19. Pascal Paillier: Public-Key Cryptosystems Based on Composite Degree Residuosity Classes. EUROCRYPT 1999: 223-238.

20. Perron: Bemerkungen über die Verteilung der quadratischen Reste, Math.Z. 56 (1952), pp.122-130.

21. John Rompel: One-Way Functions are Necessary and Sufficient for Secure Signatures, STOC 1990: 387-394.

22. Aggelos Kiayias, Moti Yung: Group Signatures with Efficient Concurrent Join. EUROCRYPT 2005: 198-214 